\title{
Effects of Spurned Help and Self-Image on Self-Defensive Behavior of Primary School Teachers
}

\author{
Kwok Sai Wong1, Wai Hing Cheuk ${ }^{2}$ \\ ${ }^{1}$ Department of Early Childhood Education, Education University of Hong Kong, Hong Kong, China \\ ${ }^{2}$ School of Arts and Social Sciences, Open University of Hong Kong, Hong Kong, China \\ Email: twong@eduhk.hk, whcheuk@ouhk.edu.hk
}

Received 27 June 2016; accepted 6 August 2016; published 9 August 2016

Copyright (C) 2016 by authors and Scientific Research Publishing Inc.

This work is licensed under the Creative Commons Attribution International License (CC BY). http://creativecommons.org/licenses/by/4.0/

(c) (i) Open Access

\section{Abstract}

How do helpers react if their offers of help have been rejected by needy others, the help has been accepted but not used, or the needy others seek help elsewhere? A model was advanced to capture how spurned helpers react (Rosen, Mickler, \& Spiers, 1986). One avenue taken to assess the validity of the model is to assess if caregiving professionals react to spurning of their help in ways consistent with the propositions of the model. Accordingly, we have been examining spurning of help by peers among schoolteachers, having explored such experiences in kindergarten and secondary school teachers. The present study extended our work to primary school teachers to test the propositions of the model through examining (a) how teachers' self-images of competence and caring influenced their reactions and (b) whether spurned teachers reacted self-defensively. 732 primary school teachers in the city of Shenzhen (China) responded to three waves of questionnaires measuring the variables under study. The results showed that the teachers experienced a moderate level of spurning, and coped by derogating the personal attributes of rejecting peers, with those having more positive self-images coping more intensely, thus supporting the validity of the propositions of the model.

\section{Keywords}

Spurn Help, Coping, Self-Image, Primary School Teachers, China

\section{Introduction}

\subsection{Overview}

How do individuals react when their help is spurned by needy others, such as their offers of help have been re- 
jected by the needy others, the help has been accepted but then not used by the help recipients, or the needy others seeking or have sought help elsewhere? As spurning of help is a form of interpersonal failure, are spurned helpers distressed and do they view themselves negatively? Do they view and relate to the rejecting others negatively? A model was advanced to capture how spurned helpers react (Cheuk \& Wong, 2004; Rosen, Mickler, \& Spiers, 1986). Subsequently, two avenues have been taken to assess the validity of the model, one being laboratory experimentation and the other being assessing whether professional caregivers react to spurning of their help in ways posited by the model. Regarding the second avenue, we have been assessing the validity of the propositions of the model through examining how spurned schoolteachers react to spurning of their help by peers and factors that influence their reactions, having examined those of secondary school teachers (Cheuk, Wong, \& Rosen, 2002) and kindergarten teachers (Cheuk, Wong, \& Rosen, 2011). Continuing this line of enquiry, the present study extended our work to the category of primary school teachers to examine, as posited by the model, whether spurned teachers would react self-defensively and whether their self-images of competence and caring influenced their reactions to being spurned. The model and related research are described below, followed by the aims and propositions of this study.

\subsection{Model and Related Research}

According to the model of spurned helpers' reactions (Cheuk \& Wong, 2004; Rosen et al., 1986), would-be helpers expect acceptance and use of their help by a needy other. Following an offer of help, however, the help may be spurned. Spurning of help, defined as thwarting of would-be helpers' intention to help or thwarting of the use of help received by the recipient, encompasses such experiences or beliefs of the helpers as (a) rejection of the offers of help by the needy others; (b) the needy others accepting the help but then not using it; or (c) the needy others seeking help elsewhere despite helpers' offers of help. The model posits that initially spurned helpers are distressed because the spurning violates their expectancy of acceptance. At this time, spurned helpers try to understand why their help was spurned. If there are indications that the spurning is unrelated to what they have done, such as because the recipient has resolved the problem, spurned helpers will no longer be distressed. However, without such indications, the unfavorable implications of spurning on spurned helpers' self-perceived competence and caring for others (i.e. the rejecter is questioning and belittling helpers' competence and caring orientation) become salient and potent, and such implications threaten spurned helpers' self-esteem. Spurned helpers then cope to protect their threatened self-esteem, such as by viewing the rejecter as too arrogant to accept or use others' help. By blaming the rejecter for the spurning, spurned helpers can view the spurning as unrelated to them, and so can still view themselves as competent and caring. Further, spurned helpers cope in multiple ways to protect self-esteem, and that their coping is influenced by personal and situational factors. The model also posits that the more frequent the occurrence of spurning, the stronger the threat to spurned helpers' self-esteem, because at higher levels of spurning, it is more difficult for spurned helpers to believe that the different incidences of spurning were unrelated to them. Rather, the negative implications from the different incidences of spurning accumulate, posing stronger threat to spurned helpers' self-esteem and motivating them to cope, with stronger threat inducing more intense coping.

To assess the validity of the model, laboratory experiments were conducted (e.g. Cheuk \& Rosen, 1992, 1996; Cheuk \& Wong, 2001, 2007) in which the help offered was actually spurned or used by others. For example, in one experiment, college students who served as tutors in a peer tutoring study witnessed a learner (a confederate) failing in two practice word-assembling tasks, then offered suggestions to help the learner do better in the ensuing test task. As pre-arranged, the help was accepted. Subsequently, some tutors "accidentally" discovered that the learner used the suggestions during the test task and some tutors found out that the learner did not use their suggestions. In another round of practice and test tasks, the tutors witnessed the learner failing again in two practice tasks, offered suggestions which were accepted, and subsequently discovered that the suggestions were or were not used. Through such and similar procedures, the reactions of spurned/accepted helpers were assessed, as were the effects of personal and situational factors. The results of these studies supported the validity of the key propositions of the model.

A second avenue that has been taken to assess the validity of the model is to examine if professional caregivers react to spurning of their help in ways consistent with the propositions of the model. As such, we examined the extent to which secondary school teachers in Guangzhou (China) experienced spurning of help by peers and how they reacted (Cheuk et al., 2002). It was predicted that at a low level of spurning, the self-esteem of the 
teachers would not be threatened, but that at a high level of spurning, the teachers would be threatened and they would cope self-protectively. We found that the teachers encountered a moderate level of spurning, and as predicted, among those who experienced higher levels of spurning, their self-esteem was threatened and they coped self-defensively by viewing rejecting peers as having negative personality attributes, such as being too arrogant to accept or use the help of others, seemingly to blame the rejecting peers for the spurning. Such results, coming from caregiving professionals working in their daily lives, support the propositions of the model regarding individuals experiencing threat to self-esteem at a high level of spurning and such individuals coping self-protectively. Subsequently, we extended our work to kindergarten teachers in Hong Kong, who were found to experience a slightly higher level of spurning than did their Guangzhou secondary school counterparts, and as predicted, were threatened by spurning, and coped similarly by derogating the personal attributes of rejecting peers (Cheuk et al., 2011).

\subsection{Objectives}

The present study had three objectives. So far, we have examined the experiences with spurning of help by peers among kindergarten and secondary school teachers (Cheuk et al., 2002, 2011) but not among primary school teachers. The first objective was to assess the extent of occurrence of spurning of help by peers among primary school teachers in a Chinese city. We believe that the levels of spurning experienced by schoolteachers in most localities depend mainly on two factors. The first one is frequency of interaction among teachers and thus the opportunities for helping peers and consequently spurning of help. Frequency of interaction is in turn contingent on the operational scale of the schools and similarity of teachers' tasks, with smaller scales of operation and higher similarity of teachers' tasks promoting more frequent interaction. The second factor is teachers' collectivistic orientation (Hofstede, 1991), with higher collectivism increasing the likelihood of helping and spurning. Given the data we have regarding extent of spurning of help by peers experienced by Guangzhou secondary school teacher, we therefore chose primary school teachers in the city of Shenzhen (China) as primary schools in Shenzhen are broadly similar to secondary schools in Guangzhou in scale of operation, type of work teachers do, and collectivistic orientation of their teachers (Lai, 2015; Ministry of Education, People's Republic of China, 2014; Van de Vliert, Yang, Wang, \& Ren, 2013; Wang \& Chen, 2010). In the light of the above similarities, we hypothesized that primary school teachers in Shenzhen would experience a moderate level of spurning as did their Guangzhou secondary school counterparts.

The second objective of the present study had to do with assessing the proposition of the model that the unfavorable implications of spurning on helpers' self-images of competence and caring threaten spurned helpers' self-esteem and such threat motivates spurned helpers to cope. In other words, this proposition indicates that helpers' self-images of competence and caring can affect how threatened they become following spurning of their help by others and how they then react.

To test the notion that helpers' self-images of competence and caring affect how threatened they become following spurning of their help by others and how they then react, a laboratory experiment has been conducted (Rosen et al., 1996). In the experiment, to understand the effects of individual differences, would-be helpers were differentiated into those high on competence and caring (high competence-high caring), those high on competence but low on caring (high competence-low caring), those low on competence but high on caring (low competence-high caring), and those low on competence and caring (low competence-low caring). It was reasoned that under a low level of spurning of help by others, the self-esteem of the four groups would not be threatened by spurning as they would be trying to understand why their help was spurned. However, under a high level of spurning of help, the high competence-high caring group would be strongly threatened and would be more threatened than would the high competence-low caring and the low competence-high caring groups, because to the first group, more significant aspects of their self-concepts would be questioned. Further, the high competence-low caring and the low competence-high caring groups would be moderately threatened and would be more threatened than the low competent-low caring group, because, for the first two groups, a significant aspect of their self-concepts would be challenged (see Table 1). The results of the experiment supported our reasoning.

In the light of the above laboratory evidence regarding the effects of self-perceived competence and caring, the self-images of competence and caring of Shenzhen primary school teachers should influence how threatened they become following spurning of their help by peers and how they then react. The second objective of the present study was to assess this. The results would shed further light on the validity of the above proposition of 
the model through the reactions of schoolteachers behaving in the daily work setting. In the present study, to understand the effects of individual differences for identifying teachers who would benefit from training regarding spurning and to follow what was done in our laboratory experiment in classifying spurned helpers (Rosen et al., 1996), we differentiated Shenzhen primary school teachers into the same four groups: high competence-high caring, high competence-low caring, low competence-high caring, and low competence-low caring. Following the reasoning adopted and results obtained in our laboratory experiment regarding the magnitudes of threat experienced by the four groups of spurned helpers (Rosen et al., 1996), we posited that under a low level of spurning of help by peers, the four groups of Shenzhen primary school teachers would not be threatened; but under a high level of spurning, the high competence-high caring group would be strongly threatened and would be more threatened than would the high competence-low caring and low competence-high caring groups; and these two groups would be moderately threatened and would be more threatened than would the low competence-low caring group (Table 2).

The model posits that the threat experienced motivates spurned helpers to cope self-protectively (Rosen et al., 1986). To assess the validity of this proposition, the third objective of the present study was to examine if spurned Shenzhen primary school teachers would react to being spurned self-protectively. Our laboratory experiments have identified various self-protective coping means exhibited by spurned helpers (e.g. Cheuk \& Wong, 2007). Thus far, our studies of schoolteachers (Cheuk et al., 2002, 2011) have focused on just one of such means of coping - viewing the rejecting peers as having negative personality attributes, doing so because this means of coping was adopted consistently by spurned helpers across our laboratory experiments. The present study would assess whether spurned Shenzhen primary school teachers would also adopt the other self-protective means of coping identified in our laboratory experiments. From the array of self-protective means of coping identified in our laboratory, we chose those that were relevant to the school setting, and these were (a) perceiving the rejecter as unsociable and (b) perceiving the rejecter as incompetent (Rosen et al., 1996). These two means of coping would be assessed in the present study, along with coping through perceiving rejecting peers as having negative personality attributes. We would also include a non-self-protective means of coping for comparison.

\subsection{Hypotheses Regarding How Shenzhen Primary School Teachers Would Cope}

One way to cope self-protectively is to view rejecting peers as having a high magnitude of negative personality attributes. Based on the magnitudes of threat posited to be experienced by the four groups of teachers under a low or high level of spurning (see Table 2) and the proposition of stronger threat inducing more intense coping, we hypothesized that under a low level of spurning, the four groups of teachers would not view rejecting peers as having high magnitudes of negative personality attributes; however, under a high level of spurning, (a) the high competence-high caring group would view rejecting peers as having a high magnitude of negative personality attributes with the magnitude being higher than those perceived by the low competence-high caring group and

Table 1. Hypothesized magnitudes of threat to self-esteem resulting from helpers' self-perceived competence and caring under a high level of help spurning.

\begin{tabular}{cccc}
\hline & & & Competence \\
\hline & & High & Low \\
\hline \multirow{2}{*}{ Caring } & High & High & Moderate \\
& Low & Moderate & Low \\
\hline
\end{tabular}

Table 2. Proposed magnitudes of threat to self-esteem resulting from teachers' self-perceived competence and caring under a high level of help spurning.

\begin{tabular}{cccc}
\hline & & & Competence \\
\hline & & High & Low \\
\hline \multirow{2}{*}{ Caring } & High & High & Moderate \\
& Low & Moderate & Low \\
\hline
\end{tabular}


the high competence-low caring group; and (b) the low competence-high caring group and the high competence-low caring group would view rejecting peers as having a moderate magnitude of negative personality attributes with the magnitude being higher than that perceived by the low competence-low caring group (see Table 3).

As spurned helpers cope in multiple ways to maximize the likelihood of protecting threatened self-esteem (Rosen et al., 1986), a second way to cope self-protectively is to view rejecting peers as incompetent. By viewing rejecting peers as incompetent and it is their incompetence that caused the spurning, such as their inabilities to realize the importance of accepting and using others' help, spurned teachers can believe that the spurning was unrelated to them and therefore can still view themselves as competent and caring. Based on the magnitudes of threat posited to be experienced by the four groups under a low or high level of spurning (see Table 2) and the proposition of stronger threat inducing more intense coping, the same pattern of coping as that of coping through derogating the personality attributes of rejecting peers was hypothesized: Under a low level of spurning, the four groups of teachers would view rejecting peers as low on incompetence; however, under a high level of spurning, the high competence-high caring group would view rejecting peers as higher on incompetence than would the low competence-high caring group and the high competence-low caring group; and these two groups would view rejecting peers as higher on incompetence than would the low competence-low caring group.

A third way to cope self-protectively is to view rejecting peers as unsociable. By viewing rejecting peers as unsociable and it is their unsociability that caused the spurning, such as their inabilities to understand the propriety of accepting help, spurned teachers can view the spurning as unrelated to them and therefore can still consider themselves as competent and caring. For this means of coping, the same pattern of coping as those of the previous two means of coping was hypothesized: Under a low level of spurning, the four groups of teachers would view rejecting peers as low on unsociability; however, under a high level of spurning, the high competence-high caring group would view rejecting peers as higher on unsociability than would the low competence-high caring group and the high competence-low caring group; and these two groups would view rejecting peers as higher on unsociability than would the low competence-low caring group.

If the proposition of the model regarding spurned helpers coping self-protectively is valid, then spurned Shenzhen primary school teachers would not blame themselves for the spurning. It was therefore hypothesized that Shenzhen primary school teachers would not blame themselves for the spurning under a low or high level of spurning.

\section{Method}

\subsection{Participants and Procedure}

In January 2015, 830 teachers from primary schools in different parts of Shenzhen were invited to participate in this study about their relationships with their peers by responding to three sets of questionnaires, all in Chinese. The first set, administered in January 2015, assessed participants' self-perceived competence and caring. The second one, administered four months afterward (May 2015), assessed participants' experiences of spurning of their help by peers in the previous months. The third set, administered two month afterward (July 2015), assessed teachers' reactions to being spurned. Informed consent regarding the study was obtained from the participants before they responded to the first set of questionnaire. After responding to the third set of questionnaire, the participants were debriefed regarding the variables studied and how the variables related to one another.

808 teachers returned the questionnaire in January 2015. Of these, 767 responded in May 2015. Of the 767 respondents, 732 returned their questionnaires in July 2015. The analyses were based on the responses of the 732 participants, most of whom were female (Male: $41 \%$; female: $59 \%$ ), fairly young (mean age $=33.34, S D=$

Table 3. Hypothesized magnitudes of negative personality attributes of rejecting peers resulting from teachers' self-perceived competence and caring under a high level of help spurning.

\begin{tabular}{cccc}
\hline & & Competence & \\
\hline & & High & Low \\
\hline \multirow{2}{*}{ Caring } & High & High & Moderate \\
& Low & Moderate & Low \\
\hline
\end{tabular}


8.89) and fairly experienced (mean years of working experience $=7.56, S D=14.43$ ). We could not recruit a representative sample due to difficulties in getting a comprehensive listing of the number and characteristics of primary schools in Shenzhen and characteristics of teachers.

\subsection{Measures}

Self-image. Self-image was assessed by a 10-item measure developed by Rosen et al., (1996), with evidence for its validity and reliability. All items were on an 11-point scale (1: applies very little to me; 11: applies very much to me). Five items assessed competence (e.g., capable). As the alpha value of the five items was acceptable (0.74), a composite was constructed by averaging the five items, with higher scores signifying higher competence. In the absence of a norm of self-conception of competence of Shenzhen primary school teachers, the mid-point of the scale of competence was used to differentiate participants into those high or low on competence. The other five items assessed a caring orientation (e.g., empathic). The alpha value of these five items was 0.78 and a composite was formed by averaging the five items, with higher scores indicating higher caring. Without a norm of self-conception of caring of Shenzhen primary school teachers, the mid-point of the scale of caring was used to differentiate participants into those high or low on caring.

Spurning of help by peers. This variable was assessed by a 12-item measure developed by Cheuk et al. (2002) with evidence for its reliability and validity. The participants rated the extent to which peer teachers spurned their help on a scale from (1) "applies very little to me” to (11) "applies very much to me” (e.g., "Peer teachers turned down my offers of help”). As the alpha value of the items was acceptable (0.80), a composite was constructed by averaging the twelve items. The mean (4.92) was used to differentiate participants into those who experienced a high or a low level of spurning.

Negative personality attributes of rejecting peers. This variable was assessed by a six-item measure developed by Rosen et al. (1996) with evidence for its reliability and validity. Using an 11-point scale (1: not at all; 11: extremely), participants rated their rejecting peers on six attributes (e.g. arrogant). As the alpha value was acceptable (0.74), a composite was formed by averaging the six items, with higher scores indicating higher magnitudes of negative personality attributes (mean $=4.43, S D=8.54)$.

Work incompetence of rejecting peers. This was assessed by a six-item measure developed by Rosen et al. (1996) with evidence for its reliability and validity. On an 11-point scale (1: not at all; 11: extremely), participants rated six work-related abilities of rejecting peers (e.g. "incapable of guiding students well”). The alpha value of the six items was 0.76 . A composite was formed by averaging the six items, with higher scores indicating higher work incompetence (mean $=3.13, S D=5.67$ ).

Unsociability of rejecting peers. This was assessed by a six-item measure developed by Rosen et al. (1996) with evidence for its reliability and validity. The participants rated the unsociability (e.g. "not knowing and respecting others' views”) of rejecting peers on an 11-point scale (1: not at all; 11: extremely). The alpha value of the items was 0.81 . A composite was formed by averaging the items, with higher scores indicating higher unsociability (Mean $=4.77, S D=7.67)$.

Regarding oneself as the cause of spurning. This variable was assessed by a 6-item measure developed for this study. On a scale from (1) “applies very little to me” to (11) "applies very much to me” (e.g., "Peer teachers did not use my help due to its ineffectiveness"), the participants rated the extent to which peer teachers spurned their help due to what the participants did. As the alpha value of the items was acceptable (0.73), a composite was constructed by averaging the six items with higher scores indicating stronger attribution of the cause of spurning to oneself (Mean $=3.15, S D=6.54)$.

\section{Results}

\subsection{Extent of Spurning}

The extent of spurning of help by peers experienced by Shenzhen primary school teachers $($ mean $=4.92)$ was similar to that of Guangzhou secondary school teachers $(t=2.35, p>.05)$, supporting our hypothesis.

\subsection{Effects of Self-Image and Spurning of Help on Coping}

As perceived negative personality attributes and perceived unsociability of rejecting peers were correlated ( $r$ $=.19, p>.05$ ), a MANOVA (Multivariate Analysis of Variance) was conducted, with spurning (high vs. low), 
competence (high vs. low) and caring (high vs. low) as the independent variables and the four means of coping as the dependent variables. The three-way interaction was significant, $F=13.89, p<.05$. As such, independent ANOVA (Analysis of Variance) was conducted for each means of coping. MANOVA and ANOVA were conducted because the independent variables were dichotomous while the dependent variables were continuous.

A three-way ANOVA was conducted with spurning of help by peers (high vs. low), competence (high vs. low) and caring (high vs. low) and as the independent variables, and perceived negative personality attributes of rejecting peers as the dependent variable. The main effects were not reported here as they were not relevant to testing the hypothesis set. The three-way interaction was significant, $F(2,714)=12.78, p<.05$. The three-way interaction was decomposed into two two-way interactions according to levels of spurning. The two-way interaction under a low level of spurning was not significant, $F(1,708)=2.87, p>.05$, with the four groups viewing rejecting peers as having low magnitudes of negative personality attributes (the means were 3.07, 3.11, 3.24 and 3.34). The two-way interaction under a high level of spurning was significant, $F(1,698)=14.21, p<.05$. Subsequent pair-wise comparisons showed that the high competence-high caring group viewed rejecting peers as having higher magnitudes of negative personality attributes (mean $=6.59$ ) than did the low competence-high caring group (mean $=5.48, p<.05$ ) and the high competence-low caring group (mean $=5.58, p<.05$ ); and these two groups viewed rejecting peers as having higher magnitudes of negative attributes than did the low competence-low caring group (mean $=4.26$, both $p s<.05$ ) (Table 4 ). The results supported our hypothesis.

A three-way ANOVA was conducted with the same three independent variables, and perceived incompetence of rejecting peers as the dependent variable. The three-way interaction was not significant, $(2,718)=4.01$, $p>.05$. The four groups of teachers viewed rejecting peers as low on incompetence under a low level of spurning (means were 2.75, 2.97, 3.06 and 3.15) or under a high level of spurning (means were 2.89, 3.07, 3.13 and 3.21). The results did not support our hypothesis.

A three-way ANOVA with the same three independent variables and perceived unsociability of rejecting peers as the dependent variable was conducted. The three-way interaction was significant, $F(2,722)=15.12$, $p<.05$. The three-way interaction was decomposed into two two-way interactions based on the two levels of spurning. Under a low level of spurning, the two-way interaction of competence and caring was not significant, $F(1,708)=2.87, p>.05$, with the four groups viewing rejecting peers as low on unsociability (means were 3.42, 3.52, 3.59 and 3.44).

Under a high level of spurning, the two-way interaction of competence and caring was significant, $F(1,714)=$ $13.87, p<.05$. Subsequent pair-wise comparisons showed that the high competence-high caring group viewed rejecting peers as higher on unsociability (mean $=6.94$ ) than did the low competence-high caring group (mean = $5.78, p<.05$ ) and the high competence-low caring group (mean $=5.93, p<.05$ ); and these two groups viewed rejecting peers as higher on unsociability than did the low competence-low caring group (mean $=4.63$, both $p s<.05$ ) (see Table 5). The results supported our hypothesis.

A three-way ANOVA was conducted with the same three independent variables, and perceiving oneself as the

Table 4. Perceived magnitude of negative personality attributes of rejecting peers resulting from teachers' self-perceived competence and caring under a high level of help spurning.

\begin{tabular}{llll}
\hline & & \multicolumn{3}{c}{ Competence } \\
\hline & & High & Low \\
\hline \multirow{2}{*}{ Caring } & High & 6.59 & 5.48 \\
& Low & 5.58 & 4.26 \\
\hline
\end{tabular}

Table 5. Perceived magnitudes of unsociability of rejecting peers resulting from teachers' self-perceived competence and caring under a high level of spurning of help.

\begin{tabular}{cccc}
\hline & & \multicolumn{2}{c}{ Competence } \\
\hline \multirow{2}{*}{ Caring } & & High & Low \\
& High & 6.94 & 5.78 \\
& Low & 5.93 & 4.63 \\
\hline
\end{tabular}


cause of spurning as the dependent variable. The three-way interaction was not significant, $(2,709)=3.45$, $p>$.05. The four groups of teachers did not view the self as the cause of spurning under a low level of spurning (means: 2.34, 2.56, 2.67 and 3.01) or a high level of spurning (means: 2.35, 2.67, 2.76 and 3.09).

\section{Discussion}

\subsection{Extent of Spurning}

Consistent with our prediction, Shenzhen primary school teachers, similar to their Guangzhou secondary school counterparts (Cheuk et al., 2002), experience a moderate level of spurning of help by peers. The levels of spurning encountered by Shenzhen primary school teachers and Guangzhou secondary school teachers, along with those experienced by Hong Kong kindergarten teachers (Cheuk et al., 2011), show the prevalence of spurning of help by peers among schoolteachers.

\subsection{Influences of Individuals' Self-Images of Competence and Caring}

The results revealed that (a) teachers high on both competence and caring coped through stronger derogation of the personality attributes and sociability of rejecting peers than did those high on either competence or caring, and (b) those high on either competence or caring coped more strongly than did those low on competence and caring. Such results show that individuals' self-images of competence and caring affect how threatened they become following spurning of their help by others and how they then react, thus lending support to the validity of the proposition of the model regarding the role of self-images of competence and caring.

The effect of self-image-higher positivity of self-image associating with stronger threat to self-esteemsuggests that positivity of self-images of spurned teachers does not buffer them from the negative effects of spurning of help. If positivity of self-image had served as a protective buffer, the spurned teachers who were high on both competence and caring, or those high on one of those two elements, would have been less threatened than would those who were low on competence and caring. Our results showed the otherwise. Research showed that individuals' high self-esteem acts as a buffer protecting the individuals from the adverse effects of task failures. For instance, compared with individuals with low self-esteem, those with high self-esteem can maintain more positive self-evaluation following failure (Heimpel, Elliot, \& Wood, 2006), and hold higher expectations of future task success (Brockner, Derr, \& Laing, 1987). Individuals with high self-esteem are more able to face failures because they have histories of task successes and positive self-views (DiPaula, Campbell, \& Diener, 2002). Spurned teachers with self-images of competence and caring should also have histories of task successes and positive self-views, but why their positive self-images do not buffer them from the negative effects of spurning awaits future investigation.

While much has been done on how teachers' self-esteem influences their reactions to successes and failures (e.g. Christou, Phillipou, \& Menon, 2001), only some attention has been paid to teachers' self-images and these studies investigated teachers’ self-images as a dependent variable (e.g. Grigorovskaya, 2000). No research has yet been done on how teachers' self-images of competence and caring influence their reactions to successes and failures on school tasks. Given the influences of self-image of competence and caring demonstrated in the present study and competence and caring being core elements of teachers' self-concepts (Zlatkovic, Stojiljkovic, Djigic, \& Todorovic, 2012), it is worth examining how teachers' self-images influence their reactions to successes and failures on school tasks, such as their reactions to negative evaluations of their teaching performance by students.

\subsection{Teachers' Coping}

That Shenzhen primary school teachers cope with spurning self-protectively through disparaging the personality of rejecting peers replicates what Hong Kong kindergarten teachers, and Guangzhou secondary school teachers do, suggesting that this means of coping is commonly adopted by schoolteachers. That Shenzhen primary school teachers also cope self-protectively through viewing rejecting peers as unsociable broadens our understanding of how schoolteachers cope. The teachers' adoption of those two means of coping, but not the means of blaming themselves for the spurning, lends support to the validity of the proposition of the model regarding spurned helpers' coping self-defensively.

Coping through disparaging the personality attributes and sociability of rejecting peers, we believe, are means 
adopted to protect threatened self-esteem. By believing that the spurning was caused not by the effectiveness of the help they offered or the way they offered help, but by peers' negative personal attributes, spurned kindergarten teachers can still view themselves as competent and caring. However, alongside this benefit, such coping appears to have two possible negative consequences. First, the spurning might have been due to the personal attributes of the rejecting peers, but it might also have been due to other reasons such as the rejecting peers considering the help not useful or the way the help was offered too intrusive. Assume that the help was spurned because the rejecting peers found the help not useful. But, believing that spurning is due to the personal attributes of the rejecting peers, spurned teachers are unlikely to explore other possible causes of the spurning. Thus, coping through derogating rejecting peers' personal attributes may hinder spurned teachers from understanding the real cause of the spurning.

Second, spurned teachers' beliefs about the negative attributes of rejecting peers are means to explain spurning to protect their self-esteem, instead of outcomes of systematic observations of rejecting peers' behavior. As such, the attributes that rejecting peers are believed to possess are unlikely to be those actually possessed by the rejecting peers. But, believing that rejecting peers possess negative personal attributes, spurned teachers are unlikely to explore further regarding what attributes such peers may have. Further, research showed that individuals are less willing to befriend those they perceive to have more negative attributes (Grayling, 2013). Research also showed that friendships carry various positive functions, such as validation of one's values (Long, 2012). Coping through derogating the personal attributes of rejecting peers therefore might lead not just to misperception of rejecting peers, but also decreased willingness to befriend those peers and lowered likelihood of experiencing the positive functions of friendships.

Contrary to our prediction, spurned teachers did not cope through devaluing the work competence of rejecting peers. One possible explanation is that rejecting peers' act of rejection implies that they are capable of dealing successfully with the problem on their own, and such an implication deters the spurned teachers from derogating their peers' competence. That spurned teachers not coping through derogating the competence of rejecting peers is inconsistent with our laboratory findings. The inconsistence is perhaps due to differences in the information about the rejecters. In the laboratory, spurned helpers could see the learner's performance on just the experimental task, whereas in the school setting, spurned teachers could see peers' performance on a variety of school tasks, including those that rejecting peers performed well, and such knowledge influenced their judgment of peers' competence.

\section{Conclusion}

Through the threat to self-esteem experienced by primary school teachers following spurning of their help by peers and how spurned teachers reacted, the validity of the propositions of the model regarding (a) the negative implications of spurning on helpers' self-images of competence and caring threatening helpers' self-esteem and (b) spurned helpers coping self-defensively was further established. While the variables in the present study were measured at different points in time, a full cross-lagged panel design should be used in future research to ascertain the posited causal relationships.

\section{References}

Brockner, J., Derr, W. R., \& Laing, W. N. (1987). Self-Esteem and Reactions to Negative Feedback: Towards Greater Generalizability. Journal of Research in Personality, 21, 318-333. http://dx.doi.org/10.1016/0092-6566(87)90014-6

Cheuk, W. H., \& Rosen, S. (1992). Helper Reactions: When Help Is Rejected by Friends or Strangers. Journal of Social Behavior and Personality, 7, 445-458.

Cheuk, W. H., \& Rosen, S. (1996). The Moderating Influence of Perceived Importance on Rejected Helpers’ Reactions. Basic and Applied Social Psychology, 18, 195-210. http://dx.doi.org/10.1207/s15324834basp1802 6

Cheuk, W. H., \& Wong, K. S. (2001). The Effects of Being Spurned and Self-Esteem on Social Worker Burnout and Coping Preference. Paper Presented at the 22nd International Conference of the Stress and Anxiety Research Society, Barcelona.

Cheuk, W. H., \& Wong, K. S. (2004). The Impacts of Outcome Importance and Self-Esteem on Teacher Burnout. Paper presented at the 25th International Conference of the Stress \& Anxiety Research Society, Amsterdam.

Cheuk, W. H., \& Wong, K. S. (2007). The Influence of Outcome Importance and Self-Esteem on Depersonalization in School Counsellors. Paper presented at the 28th International Conference of the Stress \& Anxiety Research Society, Bavaro. 
Cheuk, W. H., \& Wong, K. S., \& Rosen, S. (2002). Validation of a "Spurning Scale” for Teachers: The Chinese sample. Educational Research, 44, 289-297. http://dx.doi.org/10.1080/0013188022000031588

Cheuk, W. H., \& Wong, K. S., \& Rosen, S. (2011). The Effects of Being Spurned and Self-Esteem on Depersonalization and Coping Preferences in Kindergarten Teachers: The Case of Hong Kong. Social Psychology of Education, 14, 57-73. http://dx.doi.org/10.1007/s11218-010-9138-7

Christou, C., Phillipou, G., \& Menon, M. E. (2001). Preservice Teachers' Self-Esteem and Mathematics Achievement. Contemporary Educational Psychology, 26, 44-60. http://dx.doi.org/10.1006/ceps.1999.1028

DiPaula, A., \& Campbell, J. D. (2002). Self-Esteem and Persistence in the Face of Failure. Journal of Personality and Social Psychology, 83, 711-724. http://dx.doi.org/10.1037/0022-3514.83.3.711

Grayling, A. C. (2013). Friendship. New Haven, CT: Yale University Press.

Grigorovskaya, L. V. (2000). Many-Sidedness of the Teacher's of Music Self-Image as the Tool of an Evaluation of His Professionalism. International Journal of Psychology, 35, 249.

Heimpel, S. A., Elliot, A. J., \& Wood, J. V. (2006). Basic Personality Dispositions, Self-Esteem, and Personal Goals: Approach-Avoidance Analysis. Journal of Personality, 74, 1293-1319. http://dx.doi.org/10.1111/j.1467-6494.2006.00410.x

Hofstede, G. (1991). Cultures and Organizations: Software of the Mind. London: McGraw Hill.

Lai, L. (2015). Who Is More Collectivistic? Hong Kong Chinese or Australian Chinese. Open Journal of Social Sciences, 3 , 8-15. http://dx.doi.org/10.4236/jss.2015.39002

Long, L. D. (2012). Unchallenged, Professed Core Values: Do Undergraduate Fraternity/Sorority Members Actually Benefit in the Areas of Scholarship, Leadership, Service, and Friendship? College Student Affairs Journal, 30, 15-30.

Ministry of Education, Peoples’ Republic of China (2014). Statistics on Schools, Curriculum and Teachers. http://old.moe.gov.cn/publicfiles/business/htmlfiles/moe/s8493/index.html http://old.moe.gov.cn/publicfiles/business/htmlfiles/moe/s8493/201412/181717.html http://old.moe.gov.cn/publicfiles/business/htmlfiles/moe/s8493/201412/181621.html http://old.moe.gov.cn/publicfiles/business/htmlfiles/moe/s8494/201412/181604.html

Rosen, S., Mickler, S., \& Spiers, C. (1986). The Spurned Philanthropist. Humboldt Journal of Social Relationships, 13, 145-158.

Rosen, S., Mickler, S., Cheuk, W. H., McIntosh, W. D., Harlow, T. F., Rawa, P., \& Cochran, W. (1996). Recipient Need and Efficacious Caring as Moderators of Helpers' Reactions to Rejection and Acceptance. Current Psychology, 15, 99-112. http://dx.doi.org/10.1007/BF02686943

Van de Vliert, E., Yang, H., Wang, Y., \& Ren, X. (2013). Climato-Economic Imprints on Chinese Collectivism. Journal of Cross-Cultural Psychology, 44, 589-605. http://dx.doi.org/10.1177/0022022112463605

Wang, G., \& Chen, Y. N. K. (2010). Collectivism, Relations, and Chinese Communication. Chinese Journal of Communication, 3, 1-9. http://dx.doi.org/10.1080/17544750903528708

Zlatkovic, B., Stojiljkovic, S. D., Djigic, G., \& Todorovic, J. (2012). Self-Concept and Teachers’ Professional Roles. Social and Behavioral Sciences, 69, 377-384. http://dx.doi.org/10.1016/j.sbspro.2012.11.423

\section{Submit or recommend next manuscript to SCIRP and we will provide best service for you:}

Accepting pre-submission inquiries through Email, Facebook, LinkedIn, Twitter, etc.

A wide selection of journals (inclusive of 9 subjects, more than 200 journals)

Providing 24-hour high-quality service

User-friendly online submission system

Fair and swift peer-review system

Efficient typesetting and proofreading procedure

Display of the result of downloads and visits, as well as the number of cited articles

Maximum dissemination of your research work

Submit your manuscript at: http://papersubmission.scirp.org/ 